\title{
Perimetria computadorizada em pacientes de mais de 70 anos de idade
}

\author{
Computerized perimetry in over 70-year-old patients.
}

\author{
Maria Helena Mandello Carvalhaes ${ }^{(1)}$ \\ Rubens Belfort Jr. (2) \\ Norma Helen Medina ${ }^{(3)}$ \\ Cristiane Rolim ${ }^{(4)}$ \\ João Antonio Prata Jr. ${ }^{(5)}$
}

Estudo realizado no Departamento de Oftalmologia da Universidade Federal de São Paulo-Escola Paulista de Medicina (EPM - UNIFESP). Apoio da FAPESP.

Médico Estagiário do Setor de Glaucoma do Depto. de Oftalmologia, UNIFESP-EPM.

(2) Prof. Titular do Depto. de Oftalmologia, UNIFESPEPM.

(3) Mestre e Doutor em Oftalmologia do Depto. de Oftalmologia, UNIFESP-EPM.

(4) Mestre em Oftalmologia e Médico do Setor de Glaucoma do Departamento de Oftalmologia na UNIFESP-EPM.

(5) Mestre, Doutor e Chefe do Setor de Glaucoma do Depto. de Oftalmologia UNIFESP. Professor orientador do Curso de Pós-graduação em Oftalmologia UNIFESP-EPM.

Endereço para correspondência: Dra. Maria Helena Mandello Carvalhaes. R. Jaci, 90 apto 83, Bloco 1. São Paulo (SP). CEP 04140-080. Telefax: (011) 5717320. E-mail: mllena@bol.com.br

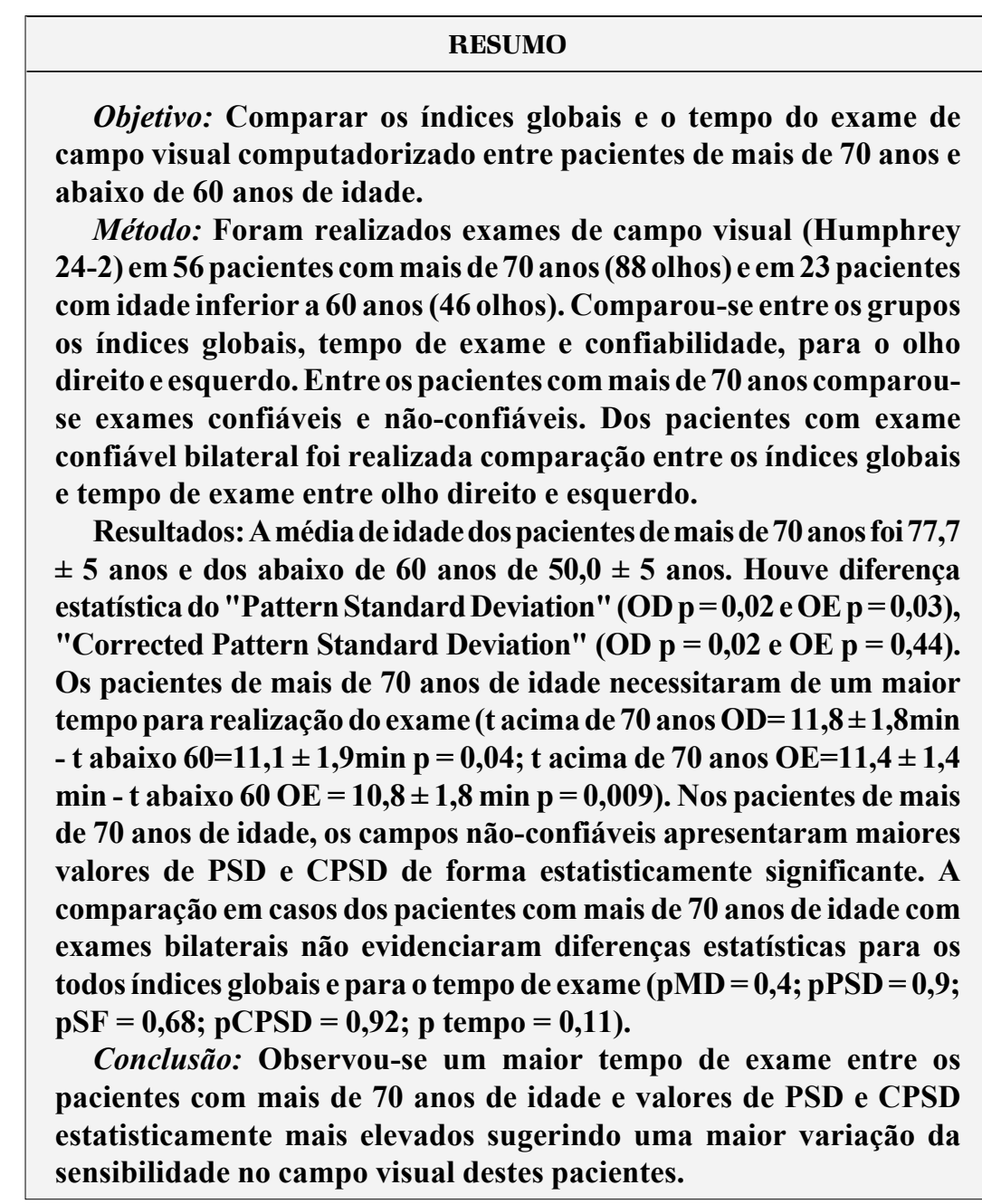

Palavras-chave: Perímetro automático Humphrey; Idade; Campo visual.

\section{INTRODUÇÃOO}

A grande vantagem do exame de campo visual computadorizado, peça importante na propedêutica oftalmológica, é eliminar a subjetividade do examinador. ${ }^{1-3}$.

Vários equipamentos estão disponíveis, mas o mais difundido é o perímetro Humphrey, que fornece um programa estatístico de análise dos resultados das médias obtidas através dos vários pontos testados (STAPAC) ${ }^{4}$. São fornecidos os índices globais para melhor compreensão dos resultados. O "Mean 
Deviation" (MD) é o desvio médio da sensibilidade do paciente se comparado com a sensibilidade média normal. O "Pattern Standard Deviation" (PSD) corresponde ao desvio padrão do gráfico "pattern deviation" e quando alterado sugere uma perda localizada de sensibilidade ${ }^{4}$. O "Short Term Fluctuation" (SF) sugere a confiabilidade das respostas do paciente durante o teste. O "Corrected Pattern Standard Deviation" (CPSD) indica a correção do PSD considerando no cálculo o valor da flutuação das respostas do paciente ${ }^{5}$.

Entretanto o exame é subjetivo e passível de influências de fatores como fadiga, colaboração, compreensão do exame, artefatos técnicos (lente corretora, pilocarpina), alterações oculares (catarata, glaucoma) e refração inadequada. Vários desses, inclusive a colaboração do paciente tendem a se agravar com a idade ${ }^{6}$.

A confiabilidade do teste pode ser observada pela análise dos índices de confiabilidade testados pelo equipamento, ou seja, fixação, respostas falso negativo e positivo. Além desses, o tempo de duração e flutuação a curto prazo elevados sugerem baixa confiabilidade, mesmo na presença de índices de confiabilidade aceitáveis ${ }^{6}$.

Bernardi e cols. ${ }^{7}$ referem que dentre aproximadamente 250 exames, $64,5 \%$ dos não confiáveis foram devido a perda de fixação. Katz e Sommer ${ }^{9}$ relatam que o excesso de perda de fixação $(67 \%$ em pacientes normais e $41 \%$ em pacientes glaucomatosos) como o principal responsável pela não confiabilidade dos exames. Keltner e col. ${ }^{11}$ estudaram o efeito de campos visuais não confiáveis no recrutamento de pacientes hipertensos oculares e encontraram, entre 10647 campos avaliados, 283 não confiáveis, sendo $73,9 \%$ destes devidos à perda de fixação.

O objetivo deste trabalho é avaliar as variações nos índices globais e tempo de exame no teste de campo visual computadorizado, nos pacientes de mais de 70 anos de idade em comparação com pacientes com menos de 60 anos de idade.

\section{MATERIAL E MÉTODO}

Foi realizado um estudo dos exames de campo visual dos pacientes com mais de 70 anos e pacientes com idade inferior a 60 anos atendidos no Departamento de Oftalmologia do Hospital São Paulo (EPM-UNIFESP). Para a inclusão no estudo, os pacientes deveriam apresentar acuidade visual maior ou igual a $20 / 40$. Foram examinados 56 pacientes acima de 70 anos de idade, 34 mulheres e 22 homens, ( 88 olhos) e 23 pacientes com idade inferior a 60 anos (46 olhos). Foram excluídos pacientes com acuidade visual menor que 20/40 ou alterações oculares que pudessem ter repercussão no campo visual, sejam alterações difusas ou localizadas (maculopatia, glaucoma). campo visual com alterações.

O campo visual foi realizado com o perímetro computadorizado Humphrey, modelo 630, com o programa 24-2, estímulo III branco. Os campos foram realizados com a refração atualizada de cada paciente.
A leitura e interpretação dos resultados dos testes foram avaliados pelo pacote de análise estatística ( STAPAC "Statistical Package"), comparando os limiares de sensibilidade do paciente e os limiares obtidos numa população normal, na mesma idade ${ }^{7-8}$.

Considerou-se como exame não-confiável a presença de perdas de fixação superiores a $20 \%$, respostas falso-positivas superiores a $33 \%$ e respostas falso-negativas superiores a $33 \%{ }^{9,10}$.

Para comparação entre os grupos selecionou-se aleatoriamente 1 olho de cada paciente. Analisou-se o tempo do exame e os índices globais pelo teste de Mann-Whitney. Dentro do grupo de pacientes com idade acima de 70 anos, procedeu-se à comparação do tempo de exame e índices globais entre exames confiáveis e não-confiáveis pelo teste de MannWhitney, empregando-se 1 olho de cada paciente escolhido ao acaso. Entre os exames confiáveis bilaterais deste grupo foi procedido a mesma comparação pelo teste de Wilcoxon.

\section{RESULTADOS}

Foram selecionados 56 pacientes acima de 70 anos e 23 pacientes com idade inferior a 60 anos. A mediana das idades dos pacientes acima de 70 foi 77 anos, média $77,7 \pm 5$ variando entre 71 a 88 anos. Destes pacientes, 42 apresentaram exames confiáveis e 14 apresentaram exames não confiáveis. Todos pacientes com idade inferior a 60 anos (23 indivíduos) apresentaram campo visual confiável e apresentaram idade média de $50 \pm 5$ anos.

Observou-se que os pacientes do grupo acima de 70 anos necessitaram de um tempo estatisticamente maior para a realização do teste tanto para o olho direito quanto para o esquerdo ( $\mathrm{t}$ geriatria ${ }_{\mathrm{OD}}=11,8 \pm 1,8$ minutos, ${ }_{\mathrm{OE}}=11,4 \pm 1,4$ minutos, $\mathrm{t}$ menores de 60 anos $_{\mathrm{OD}}=11,1 \pm 1,9$ minutos, ${ }_{\mathrm{OE}}=$ $10,8 \pm 1,8$ minutos, $\left.\mathrm{p}_{\mathrm{OD}}=0,04 \mathrm{e}_{\mathrm{OE}}=0,009\right)$.

A comparação dos índices globais revelou diferença estatisticamente significante para o índice PSD em ambos os olhos (PSD geriátrico $_{\mathrm{OD}}=2,8 \pm 1,2,{ }_{\mathrm{OE}}=2,7 \pm 1,3, \mathrm{PSD}$ menores de 60 $\left.\operatorname{anos}_{\mathrm{OD}}=2,0 \pm 0,5,_{\mathrm{OE}}=2,2 \pm 1,0, \mathrm{p}_{\mathrm{OD}}=0,002 \mathrm{e} \mathrm{p}_{\mathrm{OE}}=0,03\right)$. Nos olhos esquerdos o índice SF apresentou diferença estatisticamente marginal $(p=0,09)$ e nos olhos direitos observou-se significância para a comparação do $\operatorname{CPSD}(p=0,02)$. Não houve diferença para a comparação do MD (Tabela 1).

A principal razão para o exame não-confiável foi a perda de fixação. Entre os grupos de pacientes da geriatria com campo visual confiável e não-confiável houve diferença estatística no MD $(\mathrm{p}=0,02), \operatorname{PSD}(\mathrm{p}=0,002)$ e CPSD $(\mathrm{p}=$ 0,002) (Tabela 2).

Os pacientes do grupo confiáveis apresentaram a média de idade de 77,9 $\pm 4,8$ anos e do grupo não-confiáveis 78,9 $\pm 4,5$ anos.

Não houve diferença estatística no MD $(p=0,4)$, PSD $(p=0,9), \operatorname{SF}(p=0,7), \operatorname{CPSD}(p=0,9)$ e no tempo $(p=0,1)$, quando se comparou os olhos direito e esquerdo dos testes confiáveis dos pacientes da geriatria. 


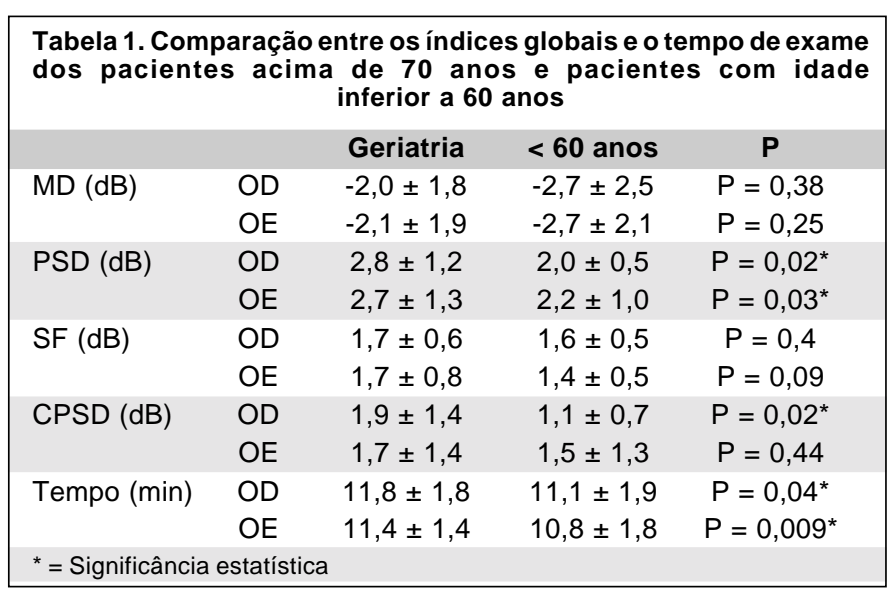

\begin{tabular}{|c|c|c|c|}
\hline & Confiável & Não confiável & $\mathbf{P}$ \\
\hline $\mathrm{MD}(\mathrm{dB})$ & $-1,9 \pm 1,8$ & $-4.1 \pm 3,4$ & $P=0,02$ \\
\hline PSD (dB) & $2,8 \pm 1,2$ & $4,9 \pm 2,4$ & $P=0,001$ \\
\hline$S F(d B)$ & $1,6 \pm 0,6$ & $1,9 \pm 0,7$ & $P=0,11$ \\
\hline CPSD (dB) & $1,9 \pm 1,5$ & $4,3 \pm 2,7$ & $P=0,002$ \\
\hline
\end{tabular}

\begin{tabular}{|c|c|c|c|}
\hline & Olho direito & Olho esquerdo & $\mathbf{P}$ \\
\hline $\mathrm{MD}(\mathrm{dB})$ & $-1,9 \pm 1,7$ & $-2,2 \pm 2,0$ & $P=0,39$ \\
\hline PSD (dB) & $2,7 \pm 1,3$ & $2,7 \pm 1,2$ & $P=0,87$ \\
\hline$S F(d B)$ & $1,7 \pm 0,5$ & $1,7 \pm 1,0$ & $P=0,68$ \\
\hline CPSD (dB) & $1,8 \pm 1,5$ & $1,7 \pm 1,3$ & $P=0,92$ \\
\hline
\end{tabular}

\section{DISCUSSÃO E CONCLUSÕES}

Foi aplicado o programa 24-2 para tentar reduzir o fator cansaço, que poderia vir a alterar os índices de confiabilidade, levando em consideração a faixa etária dos pacientes. Observou-se um tempo maior para a realização do exame em pacientes da geriatria. Isto pode ser explicado pela influência de fatores como aumento da dificuldade de compreensão do exame e fadiga que tendem a se agravar com a idade ${ }^{6}$.

O MD não mostrou diferença estatística entre o grupo da geriatria e o grupo de pacientes com idade inferior a 60 anos como era de se esperar, tendo em vista que há uma correção dos valores de sensibilidade visual para cada faixa etária ${ }^{4}$. Já a diferença estatística do PSD e CPSD entre os dois grupos sugere uma maior variação de sensibilidade nos pacientes da geriatria. Este achado sugere maior diferença de sensibilidade no campo visual pesquisado, o que talvez possa ser explicado em alterações senis.

Foram comparados os exames bilaterais dos olhos dos pacientes da geriatria para se afastar a possibilidade do efeito fadiga no $2^{\circ}$ olho examinado. Como foi utilizado apenas 1 olho de cada paciente para a comparação com o grupo de pacientes com menos de 60 anos, existia possibilidade que fossem incluídos os $2^{\mathrm{O}}$ olhos, com piora dos resultados devido a fadiga do $2^{\circ}$ olho ${ }^{4}$. Pela análise dos resultados, tal efeito pôde ser afastado. Observou-se um maior tempo de duração do exame em pacientes da geriatria e valores de PSD e CPSD estatisticamente elevados sugerindo uma maior variação de sensibilidade no campo visual.

Neste estudo, o fator mais freqüente encontrado em exames não-confiáveis foi o excesso de perda de fixação $(85,7 \%)$, o que é concordante com o apresentado por Bernardi e cols. e Katz e Sommer que referem $(65 \% \text { e } 67 \% \text { respectivamente })^{7,11}$. Ainda, foi observado uma média de tempo em exames confiáveis de 11,25 minutos. Bernardi ${ }^{7}$ e cols. estudaram 214 campos visuais de pacientes com exame confiável e encontraram a média de tempo de 15,04 minutos. Entretanto, incluímos apenas pacientes com acuidade visual $\geq 20 / 40$, sem glaucoma ou qualquer alteração grave de retina.

Observou-se nos pacientes acima de 70 anos de idade, um maior tempo de exame e valores de PSD e CPSD estatisticamente mais elevados, sugerindo uma maior variação da sensibilidade no campo visual destes pacientes.

\section{SUMMARY}

Purpose: To compare global indices and duration of automated visual field tests in geriatric patients and in patients below 60 years old.

Methods: 24-2 Humphrey visual field examinations were performed in 56 patients from the Geriatrics Department of UNIFESP-EPM and in 23 subjects aged below 60 years. Test reliability, duration and global indices were compared between groups for the right and left eyes. Among geriatric patients the same variables were studied between reliable and nonreliable fields and in those with bilateral reliable tests (OD $x O S)$.

Results: Mean age of geriatric patients was $77.7 \pm 5.0$ years and $50.0 \pm 5.0$ years in those below 60 years $(p<0.001)$. PSD and CPSD differed statistically between geriatric and nongeriatric patients (PSDOD $p=0.02$ and OS $p=0.03$; CPSDOD $p=0.02$ and $O S p=0.44$ ). Geriatric patients spent statistically more time to perform the test ( $t$ geriatrics $O D=$ $11.8 \pm 1.8 \mathrm{~min}-t$ below $60=11.1 \pm 1.9 \min p=0.04 ; \mathrm{tge}-$ riatric $O S=11.4 \pm 1.4 \mathrm{~min}-t$ below $60 \mathrm{OS}=10.8 \pm 1.8 \mathrm{~min}$ $p=0.009)$. In geriatric patients, nonreliable exams had statistically higher PSD and CPSD values than reliable fields. In those geriatric cases with bilateral exams, there were no statistical differences in global indices or test duration $(p M D=0.4 ; p P S D=0.9 ; p S F=0.68 ; p C P S D=$ 0.92; $p$ duration $=0.11$ ).

Conclusion: Geriatric patientes spent statistically more time to perform the test and had higher PSD and CPSD values, suggesting higher visual field variability.

Keywords: Humphrey perimeter; Age; Visual Field. 


\section{REFERÊNCIAS BIBLIOGRÁFICAS}

1. Wild JM, Dengler-Harles M, Searle AET, O'Neill EC, Crews JS. The influence of the learning effect on automated perimetry in patients with suspected glaucoma. Acta Ophthalmol 1989;67:537-45.

2. Birt CM, Shin DH, Samudrala V, Hugles BA, Kim C, Lee D. Anaysis of reability indices from Humphrey visual tests in a urban glaucoma population. Opthalmology 1997;104(7):1126-30.

3. Flamer I, Drance SM, Augusting L, Furkhouses A. Quantification of glaucomatous visual fields defects with automated perimetry. Invest Ophthalmol Vis Sci 1985;26:176-81.

4. Costa VP, Carvalho CA. Perimetria Computadorizada um guia básico de interpretação, $1^{a}$ edição. Rio de Janeiro: RioMed,1995, Cap.4.

5. Dias JFP. Perimetria Computadorizada, $1^{a}$ edição. Rio de Janeiro: Cultura Médica,1996, Cap.4 e 8 .
6. Johson CA, Nelson-Quigg JM. A prospective three-year study of response properties of normal subjects and patients during automated perimetry. Ophthalmology, 1993;100(2):269-74.

7. Bernardi L, Costa VP, Mutton F, José NK. Campos visuais não-confiáveis em pacientes glaucomatosos ou com suspeita de glaucoma: uma análise dos fatores de risco. Arq Bras Oftal 1999;62:57-66.

8. Enger C, Sommer A Recognizing Glaucomatous Field Loss With the Humphrey STATPAC. Arch Ophthalmol 1987;105:1355-7.

9. Katz J, Sommer A, Witt K. Reability of Visual Field Results over Repeated Testing. Ophthalmology 1991;98:70-5.

10. Bickler-Bluth M, Trick GL, Kolker AE, Cooper DG. Assessing the Utility of Reability Indices for Automated Visual Fields. Ophthalmology 1989;96:616-9.

11. Keltner JL, Johnson CA, Spurr JO, Kass MA, Gordon MO. The effect of unreliable visual fields on recruitment in the ocular hypertension treatment study. Ophthalmology 1996;103:Suppl.

\title{
I CONGRESSO BRASILEIRO DE LENTES DE CONTATO E CÓRNEA
}

\author{
12 a 14 de Abril de 2001 \\ OURO MINAS PALACE HOTEL \\ Belo Horizonte - MG
}

Promoção: Sociedade Brasileira de Lentes de Contato e Córnea - SOBLEC

Presidente de Honra: Adamo Lui Netto

Presidente: Nicomedes Ferreira Filho

Secretário: Joel Edmur Boteon

Tesoureira: Ana Luiza da S. Galeti Nehemy

Informações: Consult Comunicação e Marketing Tel./Fax: (31) 274-1550

e-mail: comunica@consultcom.com.br

internet: www.consultcom.com.br 